\title{
BMJ Open Prevalence of chronic kidney disease in adults in England: comparison of nationally representative cross-sectional surveys from 2003 to 2016
}

\author{
Hilda O Hounkpatin (D) , ${ }^{1}$ S Harris, ${ }^{1}$ Simon D S Fraser (D) , ${ }^{1}$ Julie Day, ${ }^{2}$ \\ Jennifer S Mindell (D) , ${ }^{3}$ Maarten W Taal (D) , ${ }^{4,5}$ Donal O'Donoghue, ${ }^{6}$ \\ Paul J Roderick ${ }^{1}$
}

To cite: Hounkpatin $\mathrm{HO}$, Harris S, Fraser SDS, et al. Prevalence of chronic kidney disease in adults in England: comparison of nationally representative cross-sectional surveys from 2003 to 2016. BMJ Open 2020;10:e038423. doi:10.1136/ bmjopen-2020-038423

- Prepublication history and additional material for this paper are available online. To view these files, please visit the journal online (http://dx.doi org/10.1136/bmjopen-2020038423).

Received 11 March 2020 Revised 06 May 2020 Accepted 29 May 2020

Check for updates

(C) Author(s) (or their employer(s)) 2020. Re-use permitted under CC BY-NC. No commercial re-use. See rights and permissions. Published by BMJ.

For numbered affiliations see end of article.

Correspondence to Dr Hilda 0 Hounkpatin; H.0.Hounkpatin@soton.ac.uk

\section{ABSTRACT}

Objectives To identify recent trends in chronic kidney disease (CKD) prevalence in England and explore their association with changes in sociodemographic, behavioural and clinical factors.

Design Pooled cross-sectional analysis.

Setting Health Survey for England 2003, 2009/2010 combined and 2016.

Participants 17663 individuals (aged 16+) living in private households.

Primary and secondary outcome measures Prevalence of estimated glomerular filtration rate (eGFR) $<60 \mathrm{~mL} /$ $\min / 1.73 \mathrm{~m}^{2}$ and albuminuria (measured by albumincreatinine ratio) during 2009/2010 and 2016 and trends in eGFR between 2003 and 2016. eGFR was estimated using serum creatinine Chronic Kidney Disease Epidemiology Collaboration and Modification of Diet in Renal Disease equations.

Results GFR $<60 \mathrm{~mL} / \mathrm{min} / 1.73 \mathrm{~m}^{2}$ prevalence was $7.7 \%(95 \% \mathrm{Cl} 7.1 \%$ to $8.4 \%), 7.0 \%(6.4 \%$ to $7.7 \%)$ and $7.3 \%(6.5 \%$ to $8.2 \%)$ in $2003,2009 / 2010$ and 2016 , respectively. Albuminuria prevalence was $8.7 \%$ ( $8.1 \%$ to $9.5 \%$ ) in $2009 / 2010$ and $9.8 \%(8.7 \%$ to $10.9 \%)$ in 2016 . Prevalence of CKD G1-5 (eGFR $<60 \mathrm{~mL} / \mathrm{min} / 1.73 \mathrm{~m}^{2}$ or albuminuria) was $12.6 \%$ (11.8\% to $13.4 \%$ ) in $2009 / 2010$ and $13.9 \%(12.8 \%$ to $15.2 \%)$ in 2016 . Prevalence of diabetes and obesity increased during 2003-2016 while prevalence of hypertension and smoking fell. The age-adjusted and gender-adjusted OR of eGFR $<60 \mathrm{~mL} /$ $\mathrm{min} / 1.73 \mathrm{~m}^{2}$ for 2016 versus $2009 / 2010$ was $0.99(0.82$ to 1.18 ) and fully adjusted OR was 1.13 (0.93 to 1.37). There was no significant period effect on the prevalence of albuminuria or CKD G1-5 from 2009/2010 to 2016 in age and gender or fully adjusted models.

Conclusion The fall in eGFR $<60 \mathrm{~mL} / \mathrm{min} / 1.73 \mathrm{~m}^{2}$ seen from 2003 to 2009/2010 did not continue to 2016. However, absolute CKD burden is likely to rise with population growth and ageing, particularly if diabetes prevalence continues to increase. This highlights the need for greater CKD prevention efforts and continued surveillance.

\section{INTRODUCTION}

Chronic kidney disease (CKD), defined and staged using estimated glomerular filtration

\section{Strengths and limitations of this study}

- The study is based on robust survey methodology using standardised protocols over time and taking into account complex survey design to reduce response bias.

- Prevalence may be underestimated as residential care and hospitalised patients were excluded.

- Single measures of serum creatinine and albumin to creatinine ratio so no chronicity established.

rate (eGFR) and indicators of kidney damage such as albuminuria, ${ }^{1}$ is a global public health problem with high economic cost, morbidity and mortality. ${ }^{2-4}$ The Global Burden of Disease study has shown rising global impact, largely due to population growth and ageing. ${ }^{4}$

It is important to assess trends in CKD to inform prevention and healthcare planning. There are variable data on CKD time trends. In the UK, CKD G3-5 prevalence fell in England between 2003 and 2009/2010 while remaining stable in Scotland between 2004 and $2009 / 2010 .{ }^{56}$ US studies using the National Health and Nutrition Examination (NHANES) found an increase in CKD prevalence from 1988 to 2004, followed by stabilisation between 2003/2004 and 2011/2012. ${ }^{7}$ Other studies found CKD prevalence increased in Japan from 1974 to 2002 and in Finland between 2002 and 2007; remained stable in Norway from 1995 to 2008 and decreased in Korea from 2005 to 2007. ${ }^{9-12}$ These differences in time patterns may reflect true changes, random variation or be a result of methodological and analytical differences across studies. Some projection studies have suggested an increase in CKD burden in the coming decade and beyond, which may be expected given the 
continued rise in obesity and diabetes prevalence, and ageing population. ${ }^{13-15}$

There is a lack of studies assessing CKD prevalence in recent years. It is important to examine if the earlier fall in CKD prevalence in the UK has continued and to investigate changes in albuminuria prevalence, given its prognostic importance. ${ }^{16}$ This study extends previous analyses between 2003 and 2009/2010 to examine time trends in CKD prevalence in England using the nationally representative Health Survey for England (HSE) in 2016, and to what extent any changes were explained by demographic and risk factor changes.

\section{MATERIALS AND METHODS Study population}

The HSE is an annual survey of a nationally representative sample of individuals living in private households in England. The survey, conducted by trained interviewers, collects detailed information on sociodemographic characteristics, physical health, lifestyle behaviours, mental health and well-being, and anthropometric measurements, in order to explore changes in the health and lifestyles of people in England. This is supplemented with clinical assessment by trained nurses (eg, for blood pressure (BP), medication) and with blood and urine sampling. Kidney function tests were measured in adults (aged 16+) in 2003 (serum creatinine from stored samples, 2009/2010 and 2016 (serum creatinine, cystatin $\mathrm{C}$ and urinary albumin/ creatinine ratio (ACR)).

Survey participants were selected each year using a multistage stratified probability sampling design. Full details of the methodology including sample design, response rates and weighting can be found in the 2003, 2009, 2010 and 2016 Health Survey Reports. ${ }^{17-20}$ There were household response rates of $73 \%, 68 \%, 66 \%$ and $55 \%$ for the 2003, 2009, 2010 and 2016 HSE, respectively. A total of 14836 adults were interviewed in the 2003 HSE, 4645 adults in 2009, 8420 in 2010 and 8011 in 2016.

The current study uses data from participants aged $\geq 16$ years who had valid serum creatinine or valid urine creatinine and albumin test results. The population for the current study was 17633 individuals which included $7844 / 14836(53 \%), 6053 / 13065(46 \%)$ and $3766 / 8011$ (47\%) individuals from the 2003, 2009/2010 and 2016 HSE, respectively for analyses using creatinine. Analyses for albuminuria used all available data at each time point and comprised 7633 and 4361 individuals from the 2009/2010 and 2016 HSE, respectively. Since HSE 2009 and 2010 were conducted at close time points, data from these surveys were combined to increase sample study sample size, allowing sufficient power to conduct the analyses, as was done in a previous study. ${ }^{5}$ Each survey year consisted of a new sample of participants and there was no double counting in our sample.

\section{Kidney function measures}

Serum creatinine was assayed using an isotope dilution mass spectrometry traceable enzymatic assay on a Roche Modular analyser in 2009/2010 and on a Roche Cobas analyser in 2016 in a single laboratory: the Clinical Biochemistry Department at the Royal Victoria Infirmary (RVI), Newcastle upon Tyne. Details of laboratory analysis, internal quality control and external quality assurance are provided in the 2009/2010 and 2016 documentation. ${ }^{18-20}$ The same methods were applied to the 2003 HSE samples. The 2003 HSE samples had been stored, frozen at $-40^{\circ} \mathrm{C}$ and then thawed for measurement in 2010. A correction factor was applied to 2003 samples to account for the effect of freezing on creatinine levels. ${ }^{5}$ A conversion equation derived by scientists at the RVI was then applied to the corrected 2003 creatinine values, as well as 2009 and 2010 samples, to account for differences in analysers between earlier years and 2016 and allow direct comparisons. Serum creatinine concentration was used to estimate GFR using the Chronic Kidney Disease Epidemiology Collaboration (CKDEPI) equations. ${ }^{21}$ eGFR is categorised as $<60 \mathrm{~mL} / \mathrm{min} / 1.73 \mathrm{~m}^{2}$ (CKD G3-5) and $<45 \mathrm{~mL} / \mathrm{min} / 1.73 \mathrm{~m}^{2}$ (G3b-5). The CKDEPI equation was used as this is more accurate than the Modification of Diet in Renal Disease (MDRD) equation and is recommended for use in the UK. ${ }^{22}$

Albuminuria was measured on a single random urine sample at the RVI. Urine albumin was measured by immunoassay (on a Siemens Nephelometer analyser in 2009, on a Roche Modular analyser in 2010 and on a Roche Cobas analyser in 2016). Urine creatinine was measured by colorimetric assay (Jaffe method on an Olympus analyser, Jaffe method on a Roche Modular analyser and enzymatic method on a Roche Cobas analyser in 2009, 2010 and 2016, respectively). Conversion equations (derived at the RVI) were applied to 2009 and 2010 urinary albumin and creatinine values to account for changes in analysers between 2009/2010 and 2016. Albuminuria was defined as urinary albumin to creatinine ratio (uACR) $>3 \mathrm{mg} / \mathrm{mmol}^{23}$

CKD G1-5 was defined as eGFR $<60$ and/or albuminuria and categorised as moderate, high or very high risk based on combinations of eGFR and uACR in the Kidney Disease Improving Global Outcomes (KDIGO) classification system. $^{23}$

\section{Sociodemographic characteristics}

Age was grouped into five categories: $16-34,35-54$, 55-64, 65-74 and 75+ years. Ethnicity was grouped into four categories: white, South Asian, black and other. Socioeconomic factors included occupation, car ownership and housing tenure. Occupation was classified using National Statistics Socioeconomic Classification (NS-SEC) and grouped into three categories: high (managerial and professional occupations), middle (intermediate occupations) and low (routine and manual occupations). Housing tenure was divided into 
two groups: owner and rented. Education was grouped as degree level (NVQ4/NVQ5/degree or equivalent), below degree and no qualification.

\section{Clinical and behavioural variables}

Clinical and behavioural factors included smoking (never, ex-smoker, current smoker) and body mass index (BMI; normal/underweight $\left(<25 \mathrm{~kg} / \mathrm{m}^{2}\right)$, overweight $\left(25-29.9 \mathrm{~kg} / \mathrm{m}^{2}\right)$, obese $\left(\geq 30 \mathrm{~kg} / \mathrm{m}^{2}\right)$, waist circumference (low: $<94 \mathrm{~cm}$ for men, $<80 \mathrm{~cm}$ for women; high: $94-102 \mathrm{~cm}$ for men, $80-88 \mathrm{~cm}$ women; very high: $>102 \mathrm{~cm}$ for men, $>88 \mathrm{~cm}$ for women). For South Asian men, the waist circumference was classified as: low: <90; high: $90-102$ and very high: $>102 \mathrm{~cm} .^{24}$

Clinical variables included cholesterol level (highdensity lipoprotein (HDL) and total cholesterol) from non-fasting blood samples; self-reported doctordiagnosed diabetes, survey-diagnosed diabetes (glycated haemoglobin (HBA1c) $\geq 6.5 \%$ at nurse survey examination in those not reporting a diagnosis), total diabetes (doctor + survey diagnosed); self-reported doctor-diagnosed hypertension, surveydiagnosed hypertension (systolic BP $\geq 140 \mathrm{~mm} \mathrm{Hg}$ and/ or diastolic $\geq 90 \mathrm{~mm} \mathrm{Hg}$ in those not reporting a doctor diagnosis or on medication for hypertension at the survey examination), ${ }^{19}$ and total (doctor + survey diagnosed) hypertension. Self-reported prescribed medication was any antihypertensive medication or lipid lowering agents.

Where blood pressure was not raised but antihypertensive medication data were missing, we assumed such participants did not have hypertension.

\section{Statistics}

Descriptive statistics were used to compare sociodemographic, clinical and behavioural characteristics, and kidney function measures over time, both between $2009 / 2010$ and 2016 and a trend for 2003-2016. $\chi^{2}$ tests were used to test differences for categorical variables and Somers' D rank tests were used to test for nonnormally distributed continuous variables. Normally distributed variables, including total and HDL cholesterol levels, were compared using t-tests.

Multivariable logistic regression models (including age and gender) were used to examine associations between CKD G3-5 (eGFR $<60 \mathrm{~mL} / \mathrm{min} / 1.73 \mathrm{~m}^{2}$ ) and time period (fitted as 2003, 2009/2010 and 2016), sociodemographic, clinical and behavioural variables. Similar models were applied to CKD G1-5 and albuminuria. We used complete case analysis for the primary modelling as the extent of missing data was below $10 \%$ for any variable. ${ }^{25}$ We repeated the analysis using all available data in each regression model, rather than complete case analysis.

All analyses were conducted using STATA V.14 SE and took account of the complex sampling method (including stratification and clustering due to the two-stage sampling design) and sampling weights (blood or urine as appropriate, which incorporate non-response at all stages including interview and nurse assessment to address differences in subpopulations and maintain national representativeness) through the use of the survey data commands.

Patient and public involvement (PPI)

We did not directly include PPI in this study.

\section{RESULTS}

The study population consisted of 17663 adults with serum creatinine test results and 11994 adults with valid urinary creatinine and albumin test results.

\section{Survey characteristics}

Gender and car ownership distribution were similar, while the proportion of people with older age, highest level of education, higher NS-SEC and diabetes (both doctor and survey diagnosed) increased from 2003 to 2016 (table 1). There was a decrease in the proportion of people of white ethnicity, home owners, current smokers, doctor diagnosed and total hypertension. There were increases in median levels of glycated haemoglobin, HDL cholesterol, BMI and waist circumference and decreases in systolic and diastolic BP and total cholesterol over the time period, and the changes were present from 2009/2010 to 2016 (except for diastolic BP and BMI). There was an increase in the proportion of people with doctor-diagnosed CKD between 2009/2010 and 2016, though it remained low (table 1).

\section{Kidney function measures}

Table 2 shows that there were small increases in median serum creatinine levels and concomitant decreases in eGFR levels between 2003 and 2016, largely reflected in changes in the balance of eGFR $<60-89 \mathrm{~mL} / \mathrm{min} / 1.73 \mathrm{~m}^{2}$ and $>90 \mathrm{~mL} / \mathrm{min} / 1.73 \mathrm{~m}^{2}$. eGFR $<60 \mathrm{~mL} / \mathrm{min} / 1.73 \mathrm{~m}^{2}$ prevalence was $7.7 \%$ (95\% CI $7.1 \%$ to $8.4 \%), 7.0 \%(6.4 \%$ to $7.7 \%$ ) and $7.3 \%$ (6.5\% to $8.2 \%$ ) in $2003,2009 / 2010$ and 2016, respectively. Differences were not statistically significant. There was little change in eGFR $<45 \mathrm{~mL} /$ $\min / 1.73 \mathrm{~m}^{2}$.

Figure 1 shows the pattern of eGFR $<60 \mathrm{~mL} /$ $\min / 1.73 \mathrm{~m}^{2}$ by age and gender from 2009/2010 to 2016 . Women had a higher prevalence and consistent pattern of no change in any age group. The age pattern was more variable for men.

Albuminuria prevalence was $8.7 \%(8.1 \%$ to $9.5 \%)$ in $2009 / 2010$ and $9.8 \%$ (8.7\% to $10.9 \%$ ) in 2016 . This was not statistically significant and median urinary albumin levels fell (table 2). Albuminuria prevalence increased with age with a slight $J$ shape (figure 2). In those with both urine and blood results across surveys, $88 \%$ of albuminuria was observed in people with eGFR $>60(85 \%$ in those aged 35 and over) and 16\% had doctor diagnosed diabetes (21\% in those over 35 ).

Prevalence of CKD G1-5 was $12.6 \%$ (11.8\% to $13.4 \%$ ) in 2009/2010 and $13.9 \%$ (12.8\% to $15.2 \%$ ) in 2016 (table 2). There was no significant increase in CKD 1-5 prevalence 
Table 1 Change in proportion of sociodemographic, health and lifestyle variables between 2003, 2009/2010 and 2016

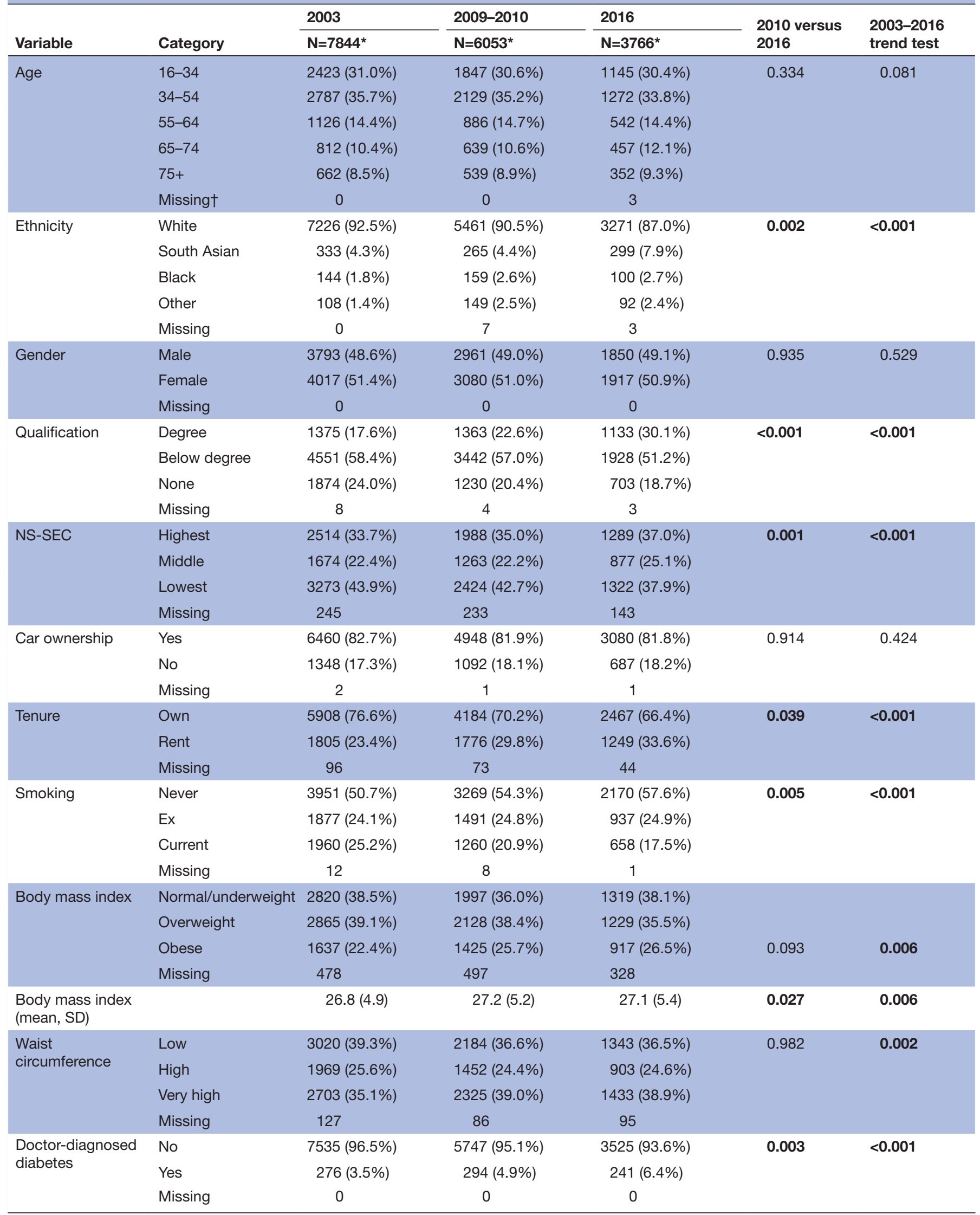

Continued 
Table 1 Continued

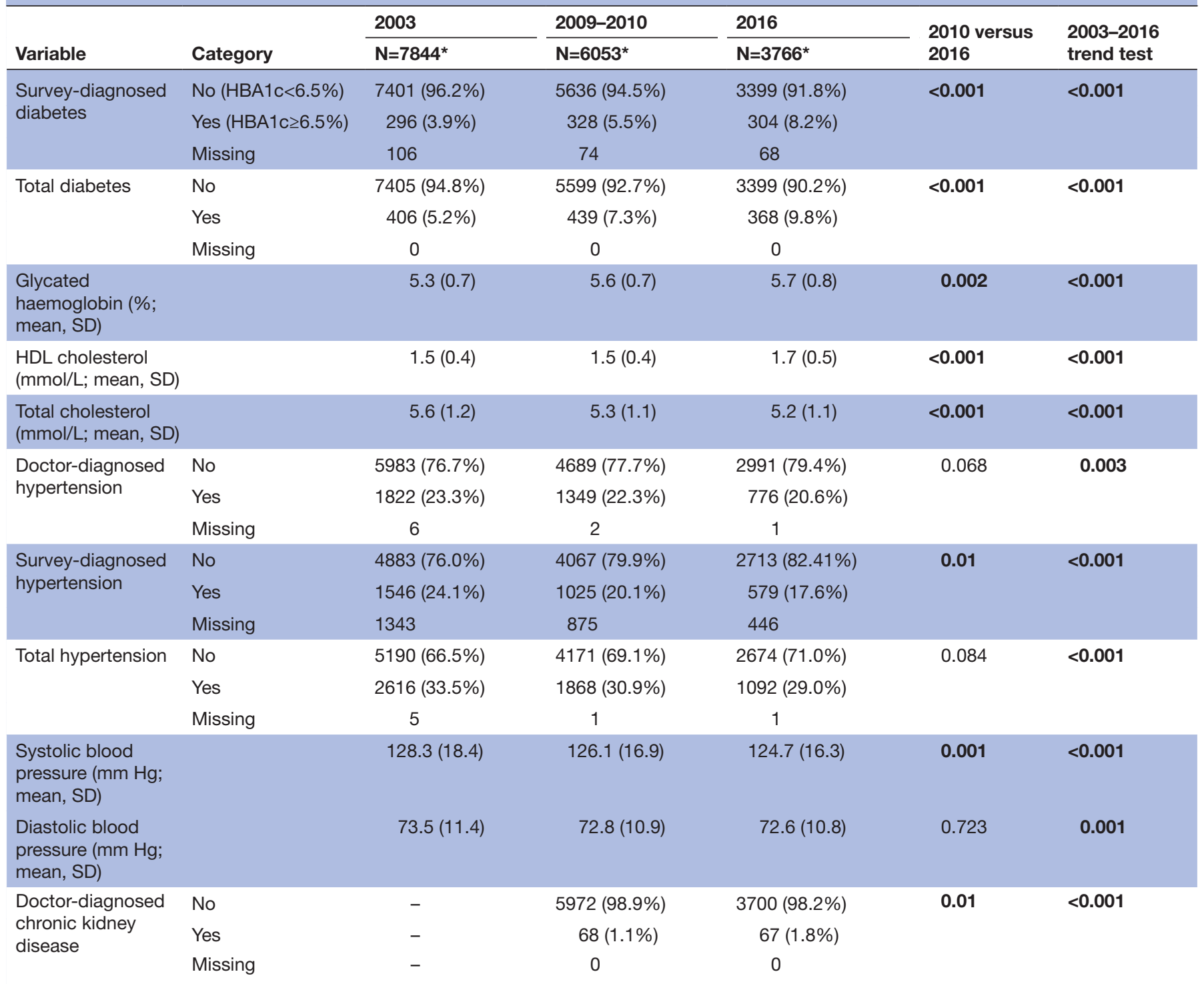

Significant $p$-values $(<0.05)$ are in bold

*Weighted to be nationally representative in each time period. Percentages are of complete data.

†Number of missing observations for each variable (not weighted).

HDL, high-density lipoprotein; NS-SEC, National Statistics Socioeconomic Classification.

overall or by any risk category, or by age group, diabetes status or obesity.

\section{Multivariable analyses}

Regression models showed no significant differences in risk of eGFR $<60 \mathrm{~mL} / \mathrm{min} / 1.73 \mathrm{~m}^{2}$ for 2016 compared with 2009/2010 (table 3 ). The OR of having eGFR $<60 \mathrm{~mL} /$ $\mathrm{min} / 1.73 \mathrm{~m}^{2}$ in 2016 compared with 2009/2010 was 0.99 (0.82 to 1.18$)$ in age- and gender-adjusted models and 1.13 (0.93 to 1.37 ) in fully adjusted models. There was no significant change in albuminuria prevalence in ageadjusted and gender-adjusted (OR: 1.05 (0.86 to 1.29)) or fully adjusted models (1.09 (0.88 to 1.36$)$; table 4$)$. The OR for having CKD 1-5 was 1.03 (0.87 to 1.21) in ageadjusted and gender-adjusted models and 1.10 (0.92 to 1.31 ) in fully adjusted models (table 5).
Sensitivity analyses using all available data for each model and outcome according to missing data found very similar results (online supplementary appendix 1). The period effect estimates in these models were consistent with the complete case models for all outcomes, though with narrower CIs due to the larger sample size and statistical power.

\section{DISCUSSION}

These nationally representative population-based studies in England have shown that the previous findings of a significant fall in CKD stage 3-5 prevalence from 2003 to $2009 / 2010$ have not continued to 2016 . There were 
Table 2 Change in renal function markers between 2003, 2009/2010 and 2016

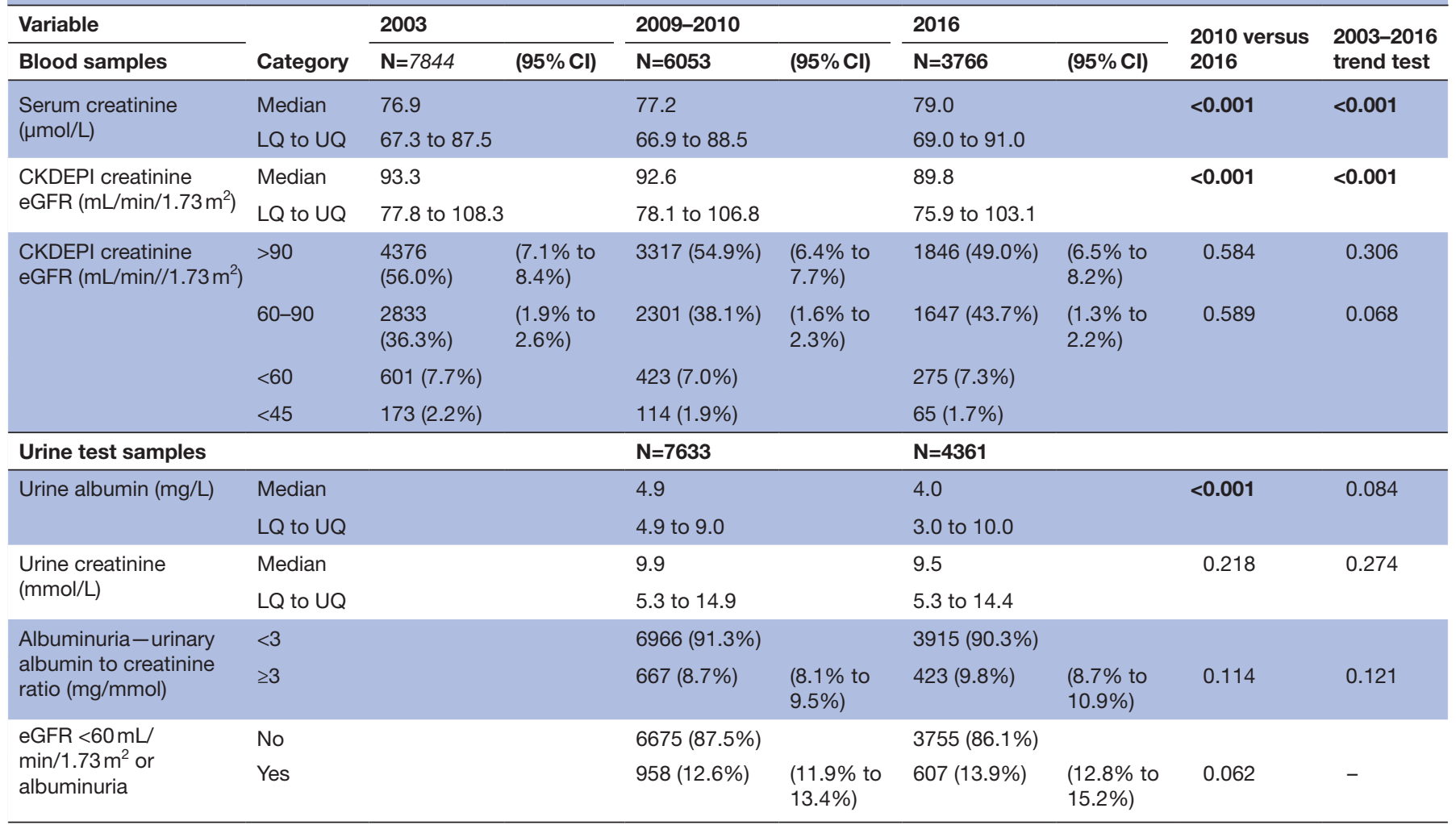

Significant $p$-values $(<0.05)$ are in bold

CKDEPI, Chronic Kidney Disease Epidemiology Collaboration; eGFR, estimated glomerular filtration rate.

no significant changes in prevalence of albuminuria and CKD G1-5 between 2009/10 and 2016.

Population risk factors for CKD changed in different directions in 2009/2010 to 2016, notably with increases in prevalence of diabetes, proportion at older age and decreases in hypertension and smoking. There were also changes in socioeconomic status and prevalence of ethnic minorities which could influence CKD prevalence. ${ }^{2627}$ The pattern of CKD prevalence is likely to reflect the balance of such countervailing CKD risk factors. Adjustment has been made for all these factors in assessing period changes in CKD prevalence.

A key finding for CKD prevention was the changes in population BP levels and hypertension prevalence which may partly be due to the decline in population salt consumption. ${ }^{28}{ }^{29}$ Changes in BP management in patients with known hypertension may also be an important factor, which we did not directly address due to limited numbers in the surveys and incomplete antihypertensive medication details. The study period coincided with the introduction in 2006 of the Quality Outcomes Framework (QoF) in England (an incentivised system for performance management of patients with diabetes, hypertension and CKD), and the National Health Service (NHS) Health Check for 40-74year olds (a national population programme with cardiovascular disease (CVD) risk factor assessment including BP measurement) ${ }^{30}{ }^{31}$ A systematic review of the impact of $\mathrm{QoF}$ on long-term conditions
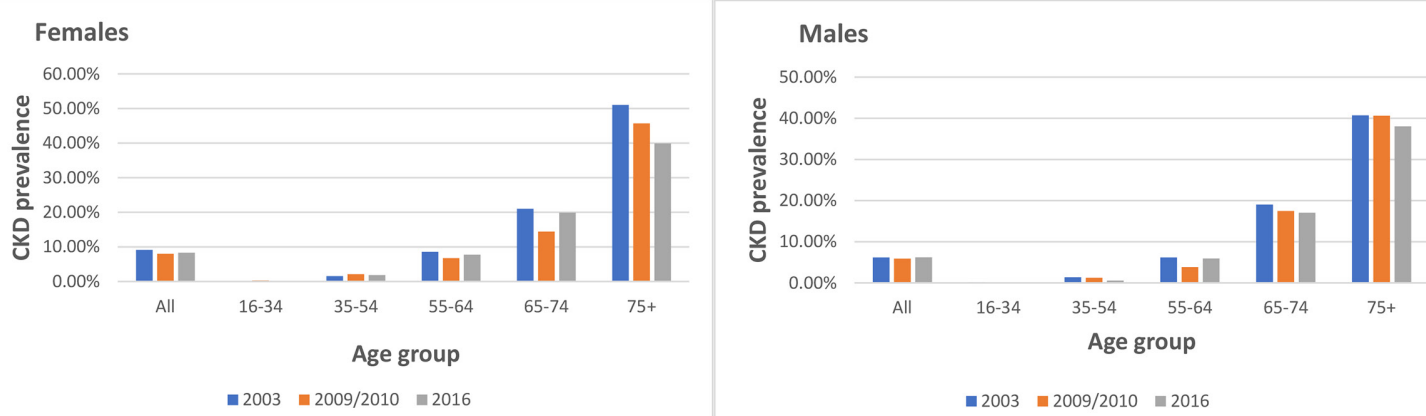

Figure 1 Changes in prevalence (weighted) of Chronic Kidney Disease (CKD) Epidemiology Collaboration serum creatinine estimated glomerular filtration rate $<60$ by age and gender 2009/2010-2016. 


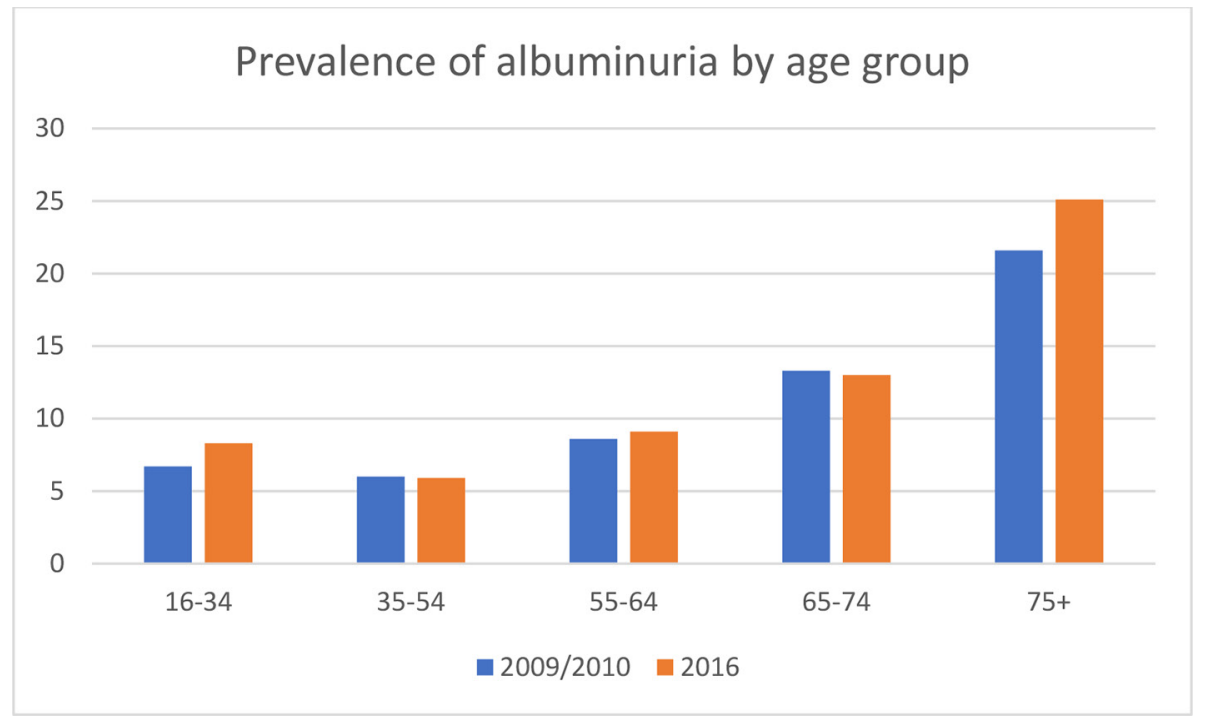

Figure 2 Changes in prevalence (weighted) of albuminuria by age group 2009/2010-2016.

found some limited evidence for improved care. ${ }^{32}$ The NHS Health Check led to small reductions in BP in nonrandomised comparisons of NHS Health Check attendees and non-attendees and to increased prescription of antihypertensive agents. ${ }^{33} 34$

The increase in HDL cholesterol and decrease in total cholesterol over time may reflect the wider use of statins for the primary and secondary prevention of CVD. HDL was associated with reduced CKD prevalence in adjusted analyses, though the effect of statins in preventing CKD progression is uncertain. ${ }^{35-37}$

A countervailing driver that would increase population CKD levels is the rising prevalence of diabetes, both diagnosed and undiagnosed. For those with diagnosed diabetes, the National Diabetes Audit for 2016-2017 found slight improvement in HBA1c control (though it was poor overall in patients with type 1 diabetes, mirroring the $\mathrm{QoF}$ review findings. ${ }^{38}{ }^{39}$ Urine ACR measurement was low in both types of diabetes and declined from 2011/2012 to $2016 / 2017$ with large variation between general practices. ${ }^{38}$ The National CKD Audit also confirmed poor recording of UACR in patients with diabetes and CKD and found it to be even poorer in those with diagnosed hypertension. ${ }^{40}$ Lack of identification of albuminuria is of concern as it is a major risk factor for both CKD progression and incident CVD and there is effective treatment available with renin-angiotensin system (RAS) inhibition. $^{41}$

To our knowledge, this study presents the most recent data on actual trends in CKD in a free-living general population. Murphy et al used serial US NHANES data from the late 1990s to 2012 and found that prevalence of CKD G3-4 and CKD G1-4 increased to the mid-2000s but then stabilised, overall and in age, gender, ethnic and diabetes subgroups except for non-Hispanic blacks. ${ }^{8}$ This was ascribed to improved management of both hypertension ${ }^{42}$ and diabetes despite rising diabetes prevalence per se. ${ }^{43}$ Hallan et al analysed the Health Survey of Nord
-Trondelag (HUNT) from 1995-1997 to 2006-2008 and found that the prevalence of CKD G1-5 was stable, which was ascribed to improved BP control and to a lesser degree to lower total cholesterol (there was no change in HDL), and greater physical activity, offset by moderate increases in obesity and diabetes. ${ }^{11}$

The survey showed that estimated CKD prevalence was substantially higher than doctor-diagnosed CKD (the latter being $1.1 \%-1.8 \%$ for $2009 / 2010$ and 2016 , respectively). This may be due to lack of diagnosis (insufficient testing in the population), or patients have not been told or cannot recall being told by their doctor that they have $\mathrm{CKD}$, as well as a small overestimation in the survey due to lack of confirmed chronicity.

\section{Study strengths and limitations}

Strengths of the study include the large, nationally representative population, as well as robust survey methodology using standardised protocols (including the same laboratory and use of conversion equations to account for changes in assays, analysers and methods over time). The complex survey design was taken into account and nonresponse blood/urine weights used to reduce response bias and ensure national representativeness. This is important, as overall HSE survey response rates have declined with time. Time trend analysis was able to take into account a range of sociodemographic, behavioural and clinical factors that were measured in a standardised way across the surveys. We were able to assess changes in uACR, which is often not measured in studies of CKD prevalence.

Limitations included the relatively short time period and fewer blood and urine samples in 2016, limiting the power of the study. Some data items were incomplete; there still is no consensus on exactly how multiple imputation should be used with survey weighted data especially when the weighting is complex and multistage as in HSE. ${ }^{44}$ Moreover, it is recommended that imputation is 


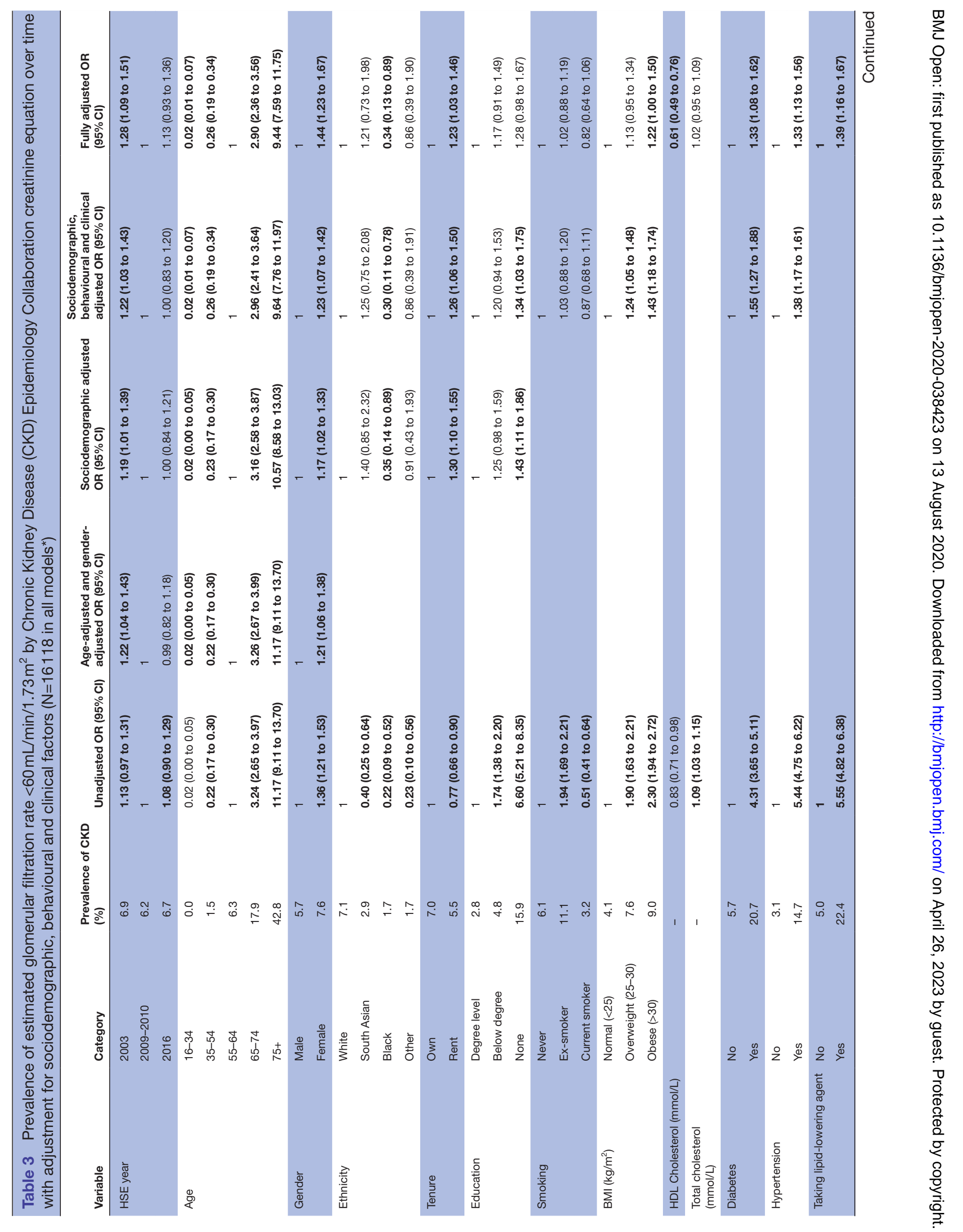


performed by the survey providers rather than secondary users. Another limitation was that the survey, as do all national health surveys, excluded those in residential care or hospitalised patients, which may include some individuals with CKD and therefore underestimates prevalence-especially of more severe CKD. On the other hand, CKD prevalence may have been overestimated as a single blood sample was tested in each survey, meaning chronicity of reduced eGFR levels could not be confirmed (as required by the KDIGO definition) and previous studies have found that fluctuations in creatinine can have a strong effect on CKD prevalence.$^{45}$ Furthermore, an isolated low eGFR could represent an episode of acute kidney injury, though this is unlikely for participants of a health survey.A single uACR is also a poor indicator of albuminuria, as data from NHANES suggest a third of initially increased uACR results may be normal if repeat testing is performed. ${ }^{7}$ ACR was measured using a random sample rather than early morning urine which may increase prevalence (orthostatic proteinuria) especially in the young, women and those without hypertension or diabetes but this should not affect trends. ${ }^{46}$ The cross-sectional nature of the survey is a limitation as it lacks chronicity and restricts the ability to infer any causal relationships from the associations identified. Although primary care databases are a suitable source of repeated measurements, testing per se and repeat testing are selective and restricted to those accessing healthcare and therefore findings may not be representative of the general population. Age was modelled as a categorical term and not linearly as data governance policy at the time of analyses precluded the availability of individual age data to download from the UK Data Archive, so given the strong effect of age it is possible that there is residual confounding. There were no data on prevalent CVD, a cause and consequence of CKD, so adjustment for trends in this was not possible. An important limitation was the lack of data on cystatin C-based eGFR trends. Estimation of GFR using an equation that includes serum creatinine and cystatin $\mathrm{C}$ gives a more accurate result than one based on creatinine alone. ${ }^{47}$ We measured cystatin $\mathrm{C}$ but the analysis suggested that differences in assay standardisation (non-standardised in 2009/2010 and standardised in 2016) accounted for a large rise observed in cystatin C concentration. The assessment of quality of care of key groups, such as those with diagnosed diabetes in HSE, was limited by numbers. There were limited data to assign likely cause(s) of CKD. Additionally, the analysis pertains to the adult population of England and may not be generalisable to other populations with different sociodemographic or underlying risk factor patterns. Finally, we used the correction factor for black ethnicity in the CKDEPI and MDRD equations to calculate eGFR. This may have introduced some bias as the correction factor was derived using US populations which may not be accurate for UK populations. However, our finding of lower prevalence of CKD in South Asian and AfricanAmericans/Afro-Caribbean compared with Caucasians is 


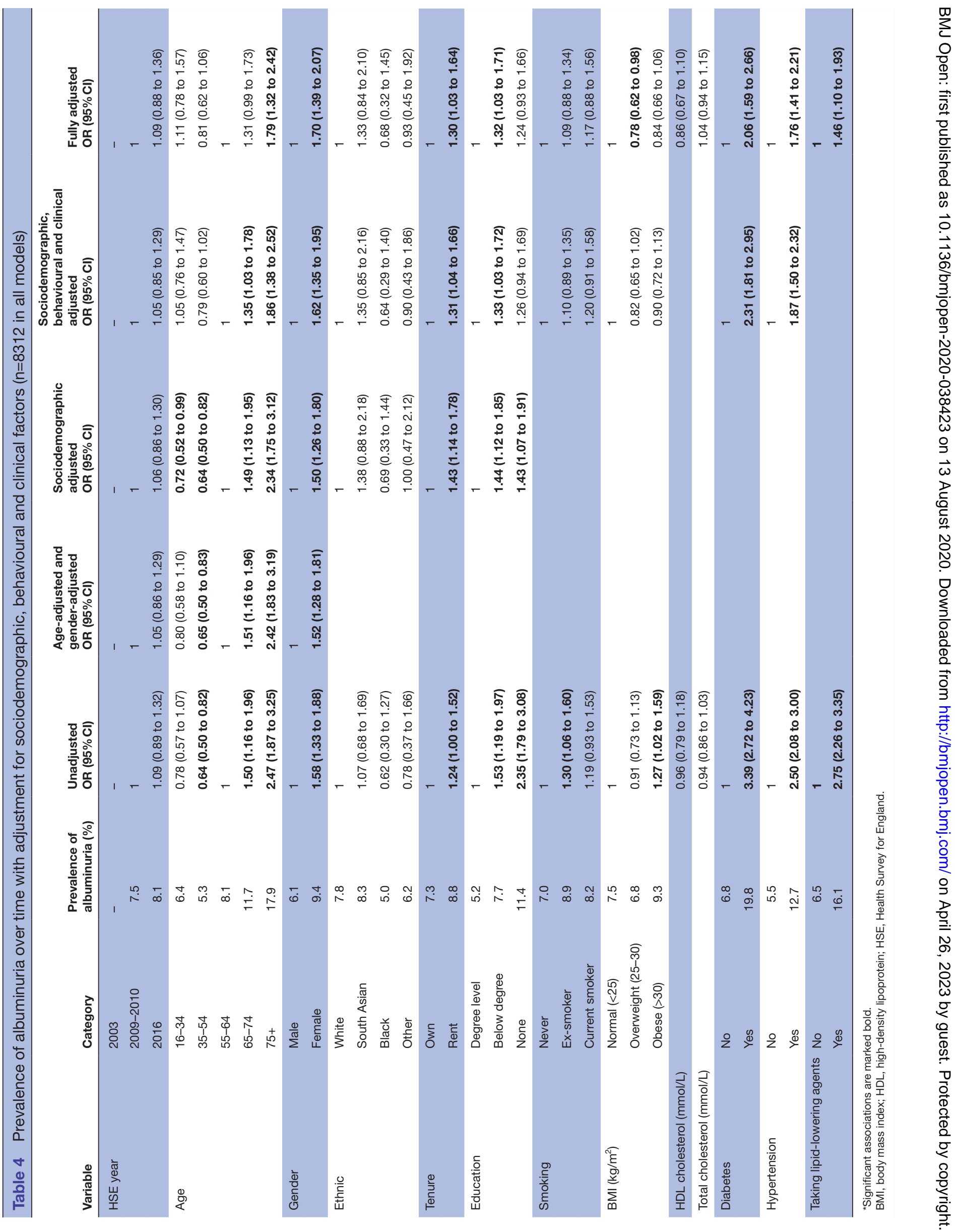




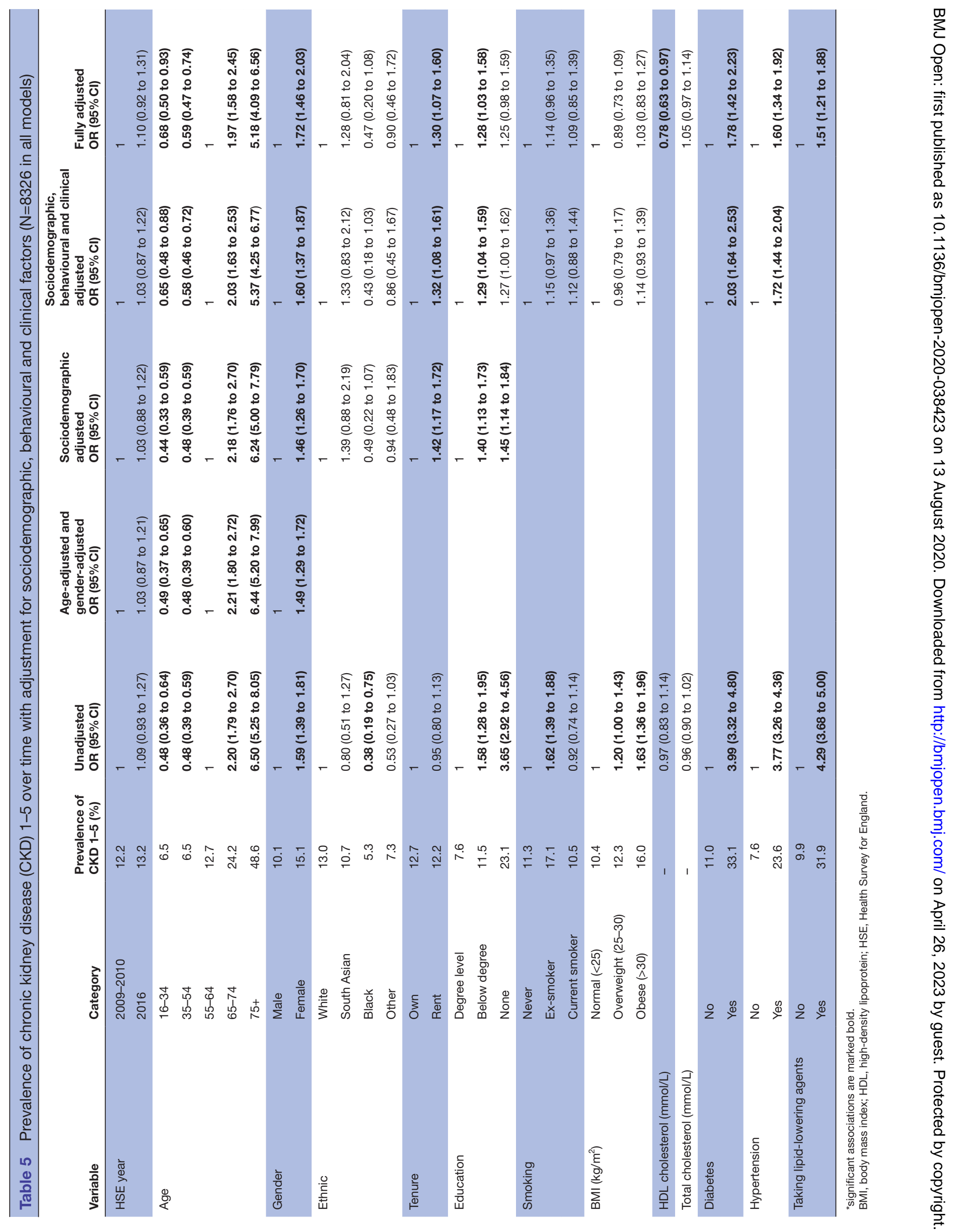


consistent with studies that report ethnic minority groups having lower or similar CKD prevalence despite higher incidence of end-stage renal disease. ${ }^{48-50}$

The finding of stable CKD prevalence may have important implications globally due to the ascending rank of CKD as a leading Global Burden of Disease cause of disability-adjusted life years and projected population growth and ageing, both of which will increase absolute CKD prevalence. ${ }^{51} 52$ Greater efforts are needed to prevent eGFR decline both in the general population and in those with CKD to reduce CKD incidence and its progression, through hypertension prevention, detection and control, obesity and diabetes prevention, and better management of existing diabetes. The underascertainment of albuminuria in patients with diabetes is of concern and merits further efforts and consideration of testing in patients with hypertension.

\section{CONCLUSION}

In this nationally representative population-based study, the previously reported trend of decreasing CKD prevalence between 2003 and 2009/2010 did not continue to 2016 despite favourable changes in hypertension prevalence. Further studies in the HSE series including using cystatin $\mathrm{C}$ and albuminuria are needed to monitor and better understand CKD trends and assess prevention efforts, and better understand mechanisms of change.

\section{Author affiliations}

${ }^{1}$ School of Primary Care, Population Sciences, and Medical Education, University of Southampton Faculty of Medicine, Southampton, UK

${ }^{2}$ Blood Sciences, Newcastle upon Tyne NHS Foundation Trust, Newcastle upon Tyne, UK

${ }^{3}$ Research Department of Epidemiology and Public Health, University College London, London, UK

${ }^{4}$ Renal Medicine, Royal Derby University Hospital NHS Foundation Trust, Derby, UK ${ }^{5}$ Centre for Kidney Research and Innovation, University of Nottingham, Derby, UK

${ }^{6}$ Nephrology, Salford Royal NHS Foundation Trust, Salford, UK

\section{Twitter Jennifer S Mindell @j_mindell}

Author contributors PR and SF conceived the original study concept and design. $\mathrm{HH}$ conducted the statistical analyses and prepared the first draft of the manuscript. SH provided guidance and advice on statistical analyses and interpretation of the data. JSM coordinated the Health Surveys for England. JD conducted the laboratory analyses. All authors critically reviewed the paper; were involved in the drafting and approval of the final manuscript; and act as guarantors. All authors take responsibility for the data and research governance.

Funding HSE 2003 was funded by the Department of Health; HSE 2009, 2010 and 2016 were funded by the Health and Social Care Information Centre (now NHS Digital). This CKD work was funded by Kidney Research UK (KRUK), grant number: KRUK RP37/2015. This funding supported kidney function testing, data management and analyses.

Disclaimer KRUK was not involved in any other aspect of the project, such as the design of the project's protocol and analysis plan, the collection and analyses. The funders had no input into the interpretation or publication of the study results.

Competing interests JSM reports grants from NHS Digital during the conduct of the study.

Patient consent for publication Not required.

Ethics approval Approval was obtained from the London Multi-Centre Research Ethics Committee for the 2003 survey (HSE 2003 ref MREC/02/2/72), the 0xford B Research Ethics Committee for both 2009 and 2010 surveys (HSE 2009 ref 08/
H0605/103, HSE 2010 ref 09/H0605/73), and the East Midlands Nottingham 2 Research Ethics Committee (Reference no 15/EM/0254) for the 2016 survey.

Provenance and peer review Not commissioned; externally peer reviewed.

Data availability statement Data are available in a public, open access repository. The HSE 2003, 2009, 2010 and 2016 are archived with the UK Data Service. The Technical Appendix, statistical code and dataset are available from the corresponding author. Creatinine measurements for the HSE 2003 undertaken for this study will be archived in due course.

Open access This is an open access article distributed in accordance with the Creative Commons Attribution Non Commercial (CC BY-NC 4.0) license, which permits others to distribute, remix, adapt, build upon this work non-commercially, and license their derivative works on different terms, provided the original work is properly cited, appropriate credit is given, any changes made indicated, and the use is non-commercial. See: http://creativecommons.org/licenses/by-nc/4.0/.

ORCID iDs

Hilda 0 Hounkpatin http://orcid.org/0000-0002-1360-1791

Simon D S Fraser http://orcid.org/0000-0002-4172-4406

Jennifer S Mindell http://orcid.org/0000-0002-7604-6131

Maarten W Taal http://orcid.org/0000-0002-9065-212X

\section{REFERENCES}

1 Eknoyan G, Lameire N, Eckardt K, et al. Kidney disease improving global outcomes (KDIGO). clinical practice guideline for the evaluation and management of chronic kidney disease. Kidney Int Suppl 2013;3.

2 Go AS, Chertow GM, Fan D, et al. Chronic kidney disease and the risks of death, cardiovascular events, and hospitalization. $N$ Engl J Med 2004;351:1296-305.

3 , Matsushita K, van der Velde M, et al, Chronic Kidney Disease Prognosis Consortium. Association of estimated glomerular filtration rate and albuminuria with all-cause and cardiovascular mortality in general population cohorts: a collaborative meta-analysis. Lancet 2010;375:2073-81.

4 GBD 2017 Disease and Injury Incidence and Prevalence Collaborators. Global, regional, and national incidence, prevalence, and years lived with disability for 354 diseases and injuries for 195 countries and territories, 1990-2017: a systematic analysis for the global burden of disease study 2017. Lancet 2018;392:1789-858.

5 Aitken GR, Roderick PJ, Fraser S, et al. Change in prevalence of chronic kidney disease in England over time: comparison of nationally representative cross-sectional surveys from 2003 to 2010 . BMJ Open 2014;4:e005480.

6 Gifford FJ, Methven S, Boag DE, et al. Chronic kidney disease prevalence and secular trends in a UK population: the impact of MDRD and CKD-EPI formulae. QJM 2011;104:1045-53.

7 Coresh J, Selvin E, Stevens LA, et al. Prevalence of chronic kidney disease in the United States. JAMA 2007;298:2038-47.

8 Murphy D, McCulloch CE, Lin F, et al. Trends in prevalence of chronic kidney disease in the United States. Ann Intern Med 2016;165:473-81.

9 Nagata M, Ninomiya T, Doi Y, et al. Trends in the prevalence of chronic kidney disease and its risk factors in a general Japanese population: the Hisayama study. Nephrol Dial Transplant 2010;25:2557-64.

10 Juutilainen A, Kastarinen $\mathrm{H}$, Antikainen R, et al. Trends in estimated kidney function: the FINRISK surveys. Eur J Epidemiol 2012;27:305-13.

11 Hallan SI, Øvrehus MA, Romundstad S, et al. Long-term trends in the prevalence of chronic kidney disease and the influence of cardiovascular risk factors in Norway. Kidney Int 2016;90:665-73.

12 Lee SW, Kim YC, Oh S-W, et al. Trends in the prevalence of chronic kidney disease, other chronic diseases and health-related behaviors in an adult Korean population: data from the Korean National health and nutrition examination survey (KNHANES). Nephrol Dial Transplant 2011;26:3975-80.

13 GBD. Risk factor Collaborators. global, regional, and national comparative risk assessment of 84 behavioural, environmental and occupational, and metabolic risks or clusters of risks for 195 countries and territories, 1990-2017: a systematic analysis for the global burden of disease study 2017. Lancet 2017;2018:1923-94.

14 Hoerger TJ, Simpson SA, Yarnoff BO, et al. The future burden of CKD in the United States: a simulation model for the CDC CKD initiative. Am J Kidney Dis 2015;65:403-11. 
15 Foreman KJ, Marquez N, Dolgert A, et al. Forecasting life expectancy, years of life lost, and all-cause and cause-specific mortality for 250 causes of death: reference and alternative scenarios for 2016-40 for 195 countries and territories. Lancet 2018;392:2052-90.

16 Turin TC, Ahmed SB, Tonelli M, et al. Kidney function, albuminuria and life expectancy. Can J Kidney Health Dis 2014;1:33.

17 Sproston K, Primatesta P. Health Survey for England 2003. In: Volume 3: methodology and documentation. London: The Stationery Office, 2004. http://webarchive.nationalarchives.gov.uk/ 20121206162012/http://www.dh.gov.uk/prod_consum_dh/groups/ dh_digitalassets/@dh/@en/documents/digitalasset/dh_4098912.pdf. (Accessed 7th August 2018).

18 Craig R, Hirani V. Health Survey for England 2009. In: Volume 2: methodology and documentation. London: The NHS Information Centre for health and social care, 2010. https://files.digital.nhs.uk/ publicationimport/pub00xxx/pub00414/heal-surv-heal-life-eng-2009rep-v3.pdf. (Accessed 7th August 2018).

19 Roth M, Roderick P, Mindell J. Kidney disease and renal function. In: Craig R, Mindell J, eds. Health survey for England 2010. Leeds: NHS Information Centre, 2011: 1-27.

20 NatCen Social Research,. University College London. In: Health survey for England 2016: methods. London: Health and Social Care Information Centre, 2017. https://files.digital.nhs.uk/publication/m/3/ hse2016-methods-text.pdf. (Accessed 7th August 2018).

21 Levey AS, Coresh J, Greene T, et al. Using standardized serum creatinine values in the modification of diet in renal disease study equation for estimating glomerular filtration rate. Ann Intern Med 2006;145:247-54.

22 National Institute of Clinical Excellence. Chronic kidney disease in adults: assessment and management. CG182. London: NICE, 2014.

23 National Kidney Foundation. KDOQI clinical practice guideline for diabetes and CKD: 2012 update. Am J Kidney Dis 2012;60:850-86.

24 National Institute for Health and Clinical Excellence. Public health draft guidance: assessing body mass index and waist circumference thresholds for intervening to prevent ill health and premature death among adults from black, Asian and other minority ethnic groups in the UK. Available: https://www.nice.org.uk/guidance/ph46/ documents/bmi-and-waist-circumference-black-and-minority-ethnicgroups-draft-guidance2[Accessed 26th January 2019].

25 Jakobsen JC, Gluud C, Wetterslev J, et al. When and how should multiple imputation be used for handling missing data in randomised clinical trials - a practical guide with flowcharts. BMC Med Res Methodol 2017;17:162.

26 Fraser SDS, Roderick PJ, Aitken G, et al. Chronic kidney disease, albuminuria and socioeconomic status in the health surveys for England 2009 and 2010. J Public Health 2014;36:577-86.

27 Dreyer G, Hull S, Aitken Z, et al. The effect of ethnicity on the prevalence of diabetes and associated chronic kidney disease. QJM 2009;102:261-9.

28 Millett C, Laverty AA, Stylianou N, et al. Impacts of a national strategy to reduce population salt intake in England: serial cross sectional study. PLoS One 2012;7:e29836.

29 Public Health England. National diet and nutrition survey results from years 1, 2, 3 and 4 (combined) of the rolling programme (2008/2009 2011/2012) 2014.

30 NHS Digital. Quality and outcomes framework, achievement, prevalence and exceptions data. Available: https://digital.nhs.uk/ data-and-information/publications/statistical/quality-and-outcomesframework-achievement-prevalence-and-exceptions-data/201718[Accessed 10thDecember 2019].

31 Nhs health check. Available: https://healthcheck.nhs.uk [Accessed 10thDecember 2019].

32 Forbes LJL, Marchand C, Doran T, et al. The role of the quality and outcomes framework in the care of long-term conditions: a systematic review. Br J Gen Pract 2017;67:e775-84.
33 Chang KC-M, Lee JT, Vamos EP, et al. Impact of the National health service health check on cardiovascular disease risk: a difference-indifferences matching analysis. CMAJ 2016;188:E228-38.

34 Martin A, Saunders CL, Harte E, et al. Delivery and impact of the $\mathrm{NHSHealthCheck} \mathrm{in} \mathrm{the} \mathrm{first} 8$ years: a systematic review. $\mathrm{Br} J \mathrm{Gen}$ Pract 2017;67:e775-84.

35 Baigent C, Landray MJ, Reith $\mathrm{C}$, et al. The effects of lowering LDL cholesterol with simvastatin plus ezetimibe in patients with chronic kidney disease (study of heart and renal protection): a randomised placebo-controlled trial. Lancet 2011;377:2181-92.

36 Heart Protection Study Collaborative Group. MRC/BHF heart protection study of cholesterol lowering with simvastatin in 20,536 high-risk individuals: a randomised placebo-controlled trial. Lancet 2002;360:7-22.

37 Haynes R, Lewis D, Emberson J, et al. Effects of lowering LDL cholesterol on progression of kidney disease. J Am Soc Nephrol 2014;25:1825-33.

38 Kontopantelis E, Reeves D, Valderas JM, et al. Recorded quality of primary care for patients with diabetes in England before and after the introduction of a financial incentive scheme: a longitudinal observational study. BMJ Qual Saf 2013;22:53-64.

39 National diabetes audit. Available: https://digital.nhs.uk/data-andinformation/clinical-audits-and-registries/national-diabetes-audit [Accessed 28th January 2019].

40 Ckd AuditNational chronic kidney disease audit: national report (Part 1 and part 2). Available: https://www.hqip.org.uk/resource/nationalchronic-kidney-disease [Accessed 28th January 2019].

41 Fraser SDS, Roderick PJ, Taal MW. Where now for proteinuria testing in chronic kidney disease?: good evidence can clarify a potentially confusing message. Br J Gen Pract 2016;66:215-7.

$42 \mathrm{Gu}$ Q, Burt VL, Dillon CF, et al. Trends in antihypertensive medication use and blood pressure control among United States adults with hypertension: the National health and nutrition examination survey, 2001 to 2010. Circulation 2012;126:2105-14.

43 Selvin E, Parrinello CM, Sacks DB, et al. Trends in prevalence and control of diabetes in the United States, 1988-1994 and 1999-2010. Ann Intern Med 2014;160:517-25.

44 Quartagno M, Carpenter JR, Goldstein H. Multiple imputation with survey weights: a multilevel approach. J Surv Stat Methodol 2019;0:1-25.

45 Eriksen BO, Ingebretsen OC. The progression of chronic kidney disease: a 10-year population-based study of the effects of gender and age. Kidney Int 2006;69:375-82.

46 Saydah SH, Pavkov ME, Zhang C, et al. Albuminuria prevalence in first morning void compared with previous random urine from adults in the National health and nutrition examination survey, 2009-2010. Clin Chem 2013;59:675-83.

47 Inker LA, Eckfeldt J, Levey AS, et al. Expressing the CKD-EPI cystatin $C$ equations for estimating GFR with standardized serum cystatin C values. Am J Kidney Dis 2011:58:682-4.

48 Xu R, Zhang L, Zhang P, et al. Comparison of the prevalence of chronic kidney disease among different ethnicities: Beijing CKD survey and American NHANES. Nephrol Dial Transplant 2009;24:1220-6.

49 USRDS: renal data system (USRDS) annual data report, Bethesda, $\mathrm{MD}$, National Institutes of health. National Institute of Diabetes and Digestive and Kidney Diseases 2008.

50 Dreyer G, Hull S, Aitken Z, et al. The effect of ethnicity on the prevalence of diabetes and associated chronic kidney disease. QJM 2009;102:261-9.

51 Jager KJ, Fraser SDS. The ascending rank of chronic kidney disease in the global burden of disease study. Nephrol Dial Transplant 2017;32:ii121-8.

52 Fraser SDS, Roderick PJ. Kidney disease in the global burden of disease study 2017. Nat Rev Nephrol 2019;15:193-4. 